\title{
Propuesta De Un Modelo De Gestión Para La Prevención De Incendios Forestales En Pinus Radiata En La Comunidad Ganquis Provincia De Chimborazo
}

\author{
Espinoza Víctor Manuel \\ Autor de la investigación Maestría Formulación, \\ Evaluación y Gerencia de proyectos para el desarrollo \\ Escuela Superior Politecnica de Chimborazo IPEC, Riobamba, Ecuador \\ Quinchuela Pozo Flor María \\ Lara Vasconez Norma Ximena \\ Espinoza Espinoza Armando Esteban \\ Miembros del tribunal Docentes Facultad Recursos Naturales \\ Escuela Superior Politecnica de Chimborazo IPEC, Riobamba, Ecuador
}

doi: 10.19044/esj.2017.v13n23p222 URL:http://dx.doi.org/10.19044/esj.2017.v13n23p222

\begin{abstract}
The objective of this study was to elaborate a management model proposal for the prevention of forest fires in the plantation of Pinus radiata located in the community of Ganquis. The approach of the management model proposal started with a forest inventory and the subsequent identification of the threats and vulnerabilities of the plantation and community. Once the information was collected, next step consisted in determining the indexes of risks to forest fires for which an adaptation was made of the methodology proposed by Sun Mountain International for the analysis of vulnerability at the municipal level raised by UNDP. The results obtained were a historical analysis of the forest fires at the province level in Chimborazo, an inventory of the volume of wood existing in the plantation and 9 potential threats and 10 vulnerabilities at the community of Ganquis and at the plantation of Pinus radiata sorrounding it. With these results the proposal of the management model for the prevention of forest fires was carried out, which has 3 guidelines that are directed to the formation of community fire brigades, prevention actions and establishment of response procedures. It is recommended that at the institutional level a system of forest fire registry be standardized in order to generate the necessary information to carry out more detailed studies on forest fires and their incidence on various natural resources.
\end{abstract}


Keywords: Threat, fire, management model, prevention, risk, vulnerability

\section{Resumen}

El objetivo de la investigación fue elaborar una propuesta de modelo de gestión para la prevención de los incendios forestales en la plantación de Pinus radiata ubicado en la comunidad de Ganquis. El planteamiento de la propuesta del modelo de gestión se inició un inventario forestal y la posterior identificación de las amenazas y vulnerabilidades de la plantación y comunidad, al contar con la información se procedió a determinar los índices de riesgos a los incendios forestales para lo cual se realizó una adaptación de la metodología propuesta por Sun Mountain Internacional para el análisis de la vulnerabilidad a nivel municipal planteado por PNUD. Los resultados obtenidos fue un análisis histórico de los incendios forestales a nivel de la provincia de Chimborazo, un inventario del volumen de madera existente en la plantación, se identificaron 9 amenazas potenciales y 10 vulnerabilidades que se encuentran presentes en la comunidad de Ganquis y la plantación del Pinus radiata. Con estos resultados obtenidos se realizó la propuesta del modelo de gestión para la prevención de los incendios forestales el mismo que cuenta con 3 líneas directrices que están dirigidas a la formación de brigadas comunitarias contraincendios, acciones de prevención y establecimiento de procedimientos de respuesta. Se recomienda que a nivel institucional se estandarice un sistema de registro de incendios forestales con el fin de generar la información necesaria para realizar estudios más detallados sobre los incendios forestales y su incidencia sobre varios recursos de naturales.

Palabras-clave: Amenaza, incendio, modelo de gestión, prevención, riesgo, vulnerabilidad

\section{Introducción}

De acuerdo a un estudio de la FAO (FAO, 2007) sobre los incendios forestales se llegó a determinar que cada año una superficie calculada en 300 millones de hectáreas sufre daños por incendios de áreas silvestres a través de un reporte de alrededor de 100.000 incendios. Esta área corresponde alrededor del $9 \%$ de la superficie forestal total, de esta superficie se ha determinado que el daño efectivo de los bosques es inferior al $5 \%$. En los últimos años muchos países han reportados el aumento del número de incendios y la gravedad de los mismos, pero estos datos no cuentan con la suficiente y la información necesaria como para concluir que el número de incendios se encuentra en aumento en el mundo. (Castillo, Pedernera, \& Peña, 2003) 
En un estudio realizado para america latina por (Manta \& León, 2004) Indica que en la región andina en los últimos 10 años se han generado una serie de incendios que han afectado en casi todas las provincias, produciendo pérdidas de varios miles de hectáreas de flora, a pesar de los esfuerzos realizados han causado daños a la fauna, aumentando el riesgo de extinción de varias especies de anfibios; además degradan recursos como el agua, suelo y aire problema que afecta directamente a las poblaciones que dependen de estos recursos (IRg/USAID/OFDALAC., 2006)

De acuerdo a la Secretaria Nacional de Gestión de Riesgos, informan que a partir del año 2008 se ha presentado una gran cantidad de incendios forestales, los mismos que se han incrementado año tras año, indistintamente de la ubicación geográfica y altitud, lo que ha obligado al gobierno a incrementar la capacidad operativa en personal y recursos para el combate de estos flagelos, de la misma manera ha generado una gran cantidad de gastos referente al equipamiento necesario para cada una de las unidades de respuesta. (Secretaria de Gestión de Riesgos, 2016)

En el año 2013 se han producido un total de 250 incendios forestales los mismos que han dejado 1200 hectáreas de plantaciones forestales comerciales y bosques nativos destruidos (FAO, 2007) la mayoría de ellos implementados con fondos de los proyectos de restauración forestal y proyectos de inversiones privadas. Por lo que es necesario el diseño de un modelo de gestión que ayudará a la prevención y reducción de riesgos de incendios forestales en base a criterios técnicos de las ciencias forestales, políticas y metodológicas de la Gestión de Riesgos a nivel del país contribuyendo a la prevención, conservación y al desarrollo sostenible de los proyectos de forestación y reforestación.

En referencia a la ubicación y características socio económicas de la zona de estudio. La misma se encuentra ubicada en la comunidad de Ganquis en la parroquia de San Juan cantón Riobamba en la zona de amortiguamiento de la Reserva de Producción Faunística de Chimborazo a una altitud de 3690 m.s.n.m (Centro de Estudios para la Gestión Publica CEGESPU, 2015). La población en esta zona se dedica al cultivo de tubérculos andinos destinados en su mayoría para la subsistencia, y pastoreo de ovejas, un gran porcentaje de los jefes de familia se dedican a trabajar como jornaleros en una las minas ubicadas en la zona perteneciente a la empresa cemento Chimborazo. (Basantes Carrillo, 2011)

\section{Materiales y métodos}

\section{Tipo de estudio y técnicas}

Se aplicó un estudio observacional en base a la recopilación y posterior análisis de la información recopilada de acuerdo a los parámetros requeridos en la plantación de Pinus radiata. 
Para el estudio se utilizó las técnicas de observación directa, recolección de información, análisis documental, simulación. Los instrumentos que facilitaron el desarrollo de la investigación y su posterior sistematización fueron: Fichas para la recolección de datos de campo, fichas de entrevistas, guía para inventario forestal, software, documentos impresos e internet.

\section{Métodos}

Para diagnosticar la situación histórica y actual de los incendios forestales, se utilizó la información existente referente a incendios forestales emitida por la Sala Situacional de la Secretaria Nacional de Gestión de Riesgos, así como del Ministerio del Ambiente. Con las bases de datos proporcionadas por las instituciones indicadas se realizó un análisis en base a Sistemas de Información Geográfica considerando ubicación, pisos altitudinales y la recurrencia de los mismos en la zona de estudio, esto con el propósito de contar con la información necesaria para realizar el análisis de riesgos.

Para determinar el grado de amenazas y vulnerabilidades se adaptó la metodología utilizada para el análisis de las amenazas, vulnerabilidades y capacidades del Ecuador, desarrollado por el instituto de investigaciones del Gobierno Francés y OXFAM Internacional en la que se señala la determinación de una escala numérica de acuerdo al peligro existente por cada uno de las zonas y rodales de la plantación siendo la zona I la de menor peligro y la zona IV la de mayor peligro, se procede a calificar de 0 como un valor mínimo y 3 como un máximo. En el caso del análisis del riesgo se realizó una adaptación de la propuesta metodológica de Sun Mountain Internacional para el análisis de vulnerabilidad física a nivel municipal con escalas numéricas que van de $1-10$.

En el cálculo del índice riesgo se utilizó el criterio analítico o matemático el cual se fundamenta en la aplicación de la siguiente ecuación $\mathrm{R}=\mathrm{A} * \mathrm{~V}$ planteada por (Estrategia Internacional para la Reducción de Desastres, 2009), donde $\mathrm{R}$ es el riesgo, A es la amenaza y $\mathrm{V}$ es la vulnerabilidad; esta ecuación es la referencia básica para la estimación del riesgo en donde cada una de las variables las expresamos en términos de probabilidad. Además se determinó el grado de recurrencia y estacionalidad de los incendios forestales en la zona.

Para generar la propuesta de prevención de incendios se utilizaron los valores obtenidos en el cálculo del índice del riesgo generando una hoja de cálculo que permitió clasificar y sistematizar las amenazas que influyen directamente en los incendios forestales. Esto permitió identificar de forma clara y numérica los tipos de eventos que pueden generar los incendios, el origen de los mismos y como se relacionan entre ellos. 


\section{Resultados y discusión}

Situación histórica y actual de los incendios forestales.

En relación a la situación de registros formales de incendios forestales se determina que a partir de los años 1960 se comienza a registrar estos eventos en Chile, en España existen registros desde 1844 publicado en el Boletín Oficial de la Provincia de Santander (Guitián Rivera, 1999). Mientras que en países como Alemania que históricamente su economía se ha basado en el cultivo de bosques, existen registros de incendios a partir del siglo XVIII.

Al realizar el análisis sobre la situación histórica de los incendios forestales registrados oficialmente en la provincia de Chimborazo, tenemos que esta información la podemos encontrar a partir del año 2011, hay que mencionar que desde el 2008 se generó un gran número de incendios a nivel del territorio pero no ha sido registrada esta información de forma oficial por las instituciones responsables como lo podemos observar en el grafico 1, que se registra un evento para el año 2011. A partir del año 2012 se comienza a registrar los incendios de forma sistémica por parte de la Secretaria de Gestión de Riesgos y del Ministerio del Ambiente, de la misma forma se empieza a ingresar la información al sistema de inventarios de desastres y eventos adversos DESINVENTAR.

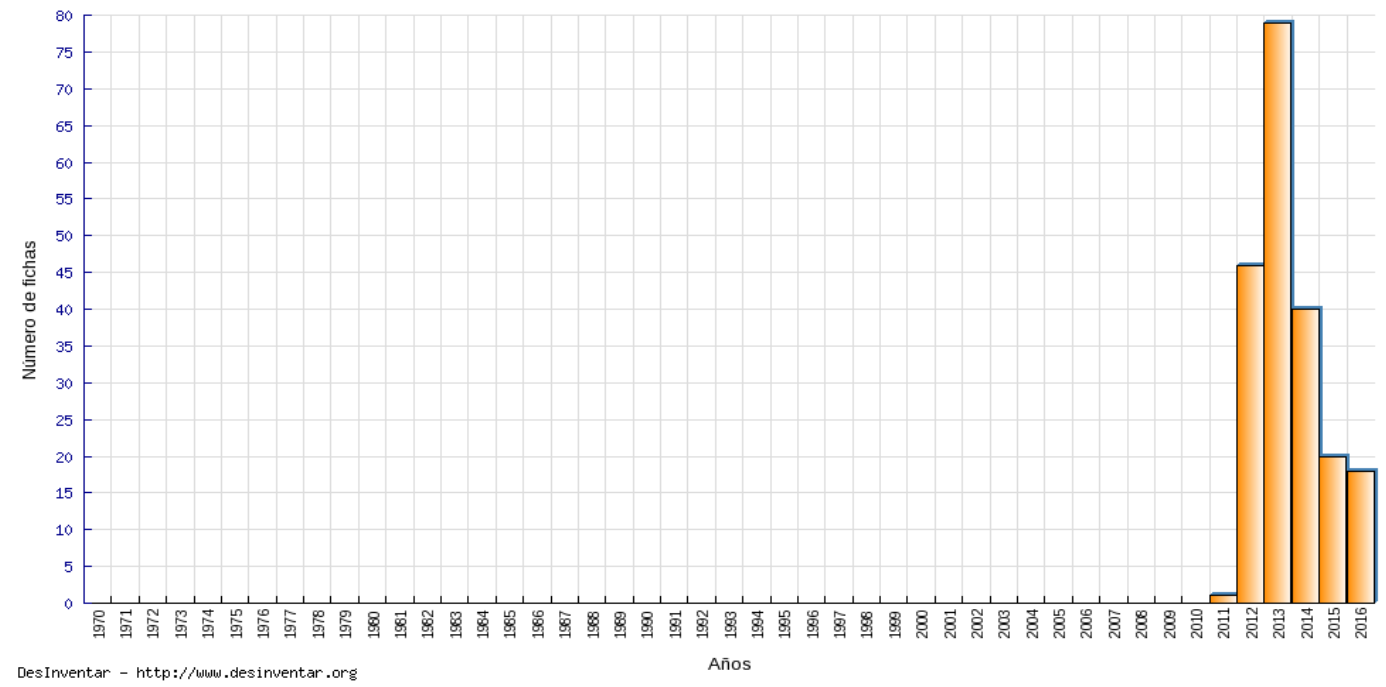

Figura 1. Registro histórico de incendios forestales en la provincia de Chimborazo Fuente: Proyecto DESINVENTAR

Con referencia a los incendios ocurridos en el año 2012 se registran un total de 25 eventos, de los cuales 13 se encuentran en el cantón Colta y 7 incendios en el cantón Pallatanga. En el año 2013 el mayor número de incendios se suscitaron en el cantón Colta, reportándose un total de 6 incendios y en segundo lugar el Cantón Riobamba con 5 incendios, en menor 
proporción se presentan en los cantones Chambo con 3, Guamote 2 , Cumandá y Guano con 1 en cada cantón.

En el año 2015 el total de incendios es de 21 eventos, de los cuales más del $50 \%$ de estos corresponde al cantón Riobamba con 13 incendios, y en menor proporción se presenta en los cantones de Guamote y Guano con 3 en cada uno. En los cantones de Chambo y Colta se registró 1 evento en cada uno.

En el año 2016 en un número total de incendios forestales en la provincia de Chimborazo es 54, los mismos los mismos que se encuentran distribuidos en 8 cantones excepto los cantones de Cumandá y Chambo. El mayor número de incendios se presentan en los cantones de Colta con 14 eventos, Guamote con 11 y Riobamba con 10 eventos.

Realizando el análisis de los incendios forestales entre los años 2012 al 2016 referente a su ubicación encontramos que menos del $1 \%$ se encuentra dentro del área de amortiguamiento de la Reserva de Producción Faunística Chimborazo, ya para el año 2013 se registra un total de 11,42\% dentro de la Reserva y un 10,89 \% en la zona de amortiguamiento. En el año 2014 aumentan los eventos llegando al 30.89\% dentro de la Reserva del Chimborazo y reduciéndose al $4 \%$ en la zona de amortiguamiento.

De acuerdo a los reportes del MAE y SGR en la plantación forestal en Ganquis se reportaron 6 conatos de incendios forestales, dos eventos que se registran durante el año 2015 tienen como particularidad una duración de 5 y 7 días respectivamente; en el evento correspondiente al 7 de septiembre se registra un daño parcial en la plantación de Pinus radiata mientras que la mayor parte de afectación corresponde a pajonal existente en la zona de acuerdo a los informes técnicos proporcionados por el Ministerio del Ambiente para el presente estudio.

\section{Vulnerabilidades, amenazas e índices de riesgos para incendios forestales en la plantación de Pinus radiata en Ganquis}

El estudio se lo realizó en cada uno de los 3 rodales existentes en la plantación El rodal 1 presenta un área de 37 hectáreas con árboles que cuentan con las características necesarias para la producción de madera de calidad, en la parte intermedia se ubica el rodal 2 con 38 hectáreas el mismo que presenta árboles con medianas características para la producción de madera, mientras que en el rodal 3 se encuentra en la parte más alta de la plantación ubicada entre los 3900 a 4100 m.s.n.m rodal en el cual los árboles casi no se han desarrollado y la madera que se puede extraer en esta zona corresponde a madera de tercera calidad.

Al realizar un cálculo global del costo de los 3 rodales tenemos que el valor de la plantación de Pinus radiata para el volumen comercial es de 231.484,18 dólares y un valor de 76.363,60 dólares para el volumen de 
madera de tercera calidad, Con un valor total de $307.847,78$ dólares en toda la plantación.

Las amenazas se las determinó de acuerdo a los resultados de casos de los incendios forestales que fueron investigados a nivel del país, debido a que en la mayoría de los reportes de los incendios en la zona de estudio se registra como causa desconocida. En los últimos años se llegaron a investigar un total de 175 incendios forestales de los cuales el $47 \%$ fueron causados intencionalmente en lugares poco accesibles, el $25 \%$ fueron causados por quema de la basura, las quemas agrícolas corresponde al 14\%, niños jugando con fuego con el fin de atrapar conejos el $7 \%$, el $4 \%$ por causa de los fumadores y por último el $3 \%$ debido a las fogatas dejadas por los turistas.

\section{CARACTERIZACION DE LA VULNERABILIDAD}

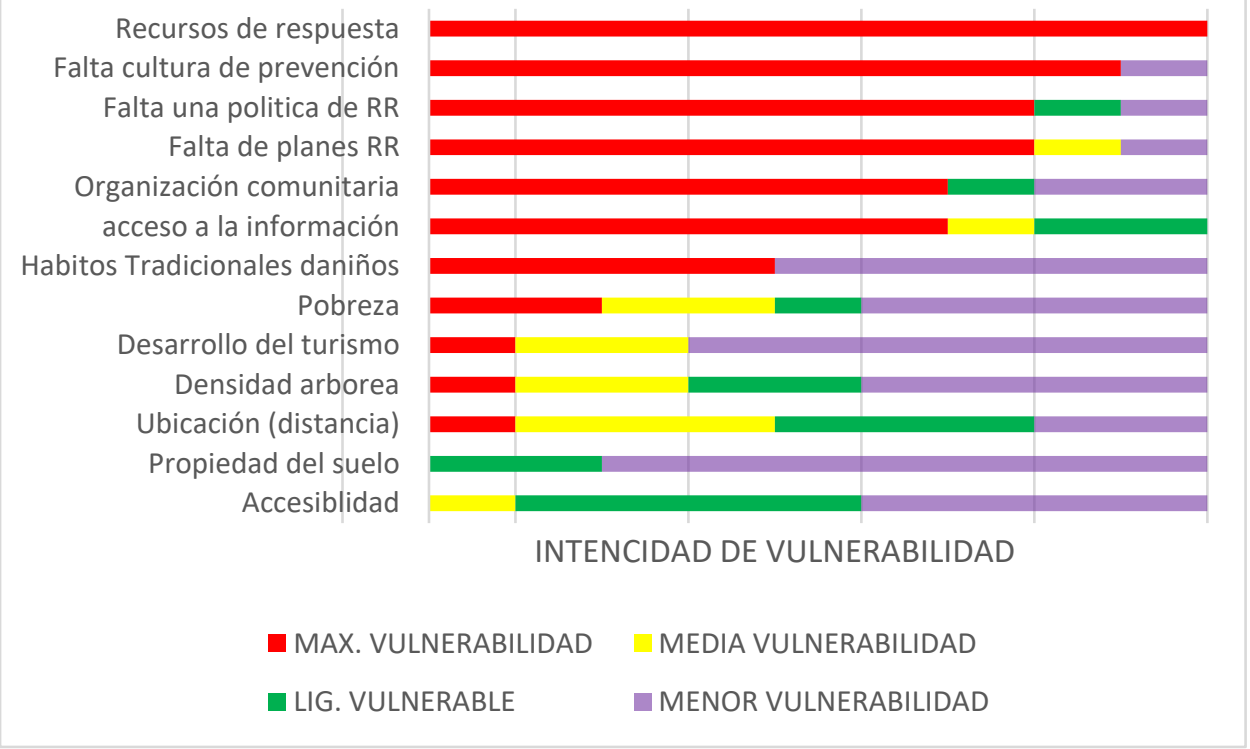

Figura: 2: Caracterización de las vulnerabilidades Elaborado: Víctor Espinoza, 2016

En la figura 2 se procedió a sintetizar de forma ordenada cada una de los factores de vulnerabilidades existentes en la zona considerando la de mayor influencia hasta las que tienen una menor incidencia. Determinándose que 11 factores son los que generan una máxima vulnerabilidad de los cuales 6 presentan una gran influencia como la falta de recursos de respuesta, ausencia de una cultura de prevención, el que no se aplique una política de reducción de riesgos, no contar con planes de reducción de riesgos, no hay una organización comunitaria respecto a los riesgos existentes en la comunidad y por último el limitado acceso a la información. 
Tabla: 1 Calculo del riesgo a incendios forestales

\begin{tabular}{|c|c|c|c|c|c|c|c|c|c|c|}
\hline VULNER & BILIDAD & $\begin{array}{c}\text { Piro } \\
\text { maniático } \\
\mathbf{S} \\
\text { (intencion } \\
\text { ales) } \\
\end{array}$ & $\begin{array}{c}\text { Quemas } \\
\text { regenerac } \\
\text { ión de } \\
\text { pasto }\end{array}$ & $\begin{array}{c}\text { Quem } \\
\text { as } \\
\text { agrícol } \\
\text { as }\end{array}$ & $\begin{array}{c}\text { Que } \\
\text { ma } \\
\text { de } \\
\text { basur } \\
\text { a } \\
\end{array}$ & $\begin{array}{c}\text { Que } \\
\text { ma } \\
\text { para } \\
\text { caza }\end{array}$ & $\begin{array}{c}\text { Fumado } \\
\text { res }\end{array}$ & $\begin{array}{c}\text { Ray } \\
\text { os }\end{array}$ & $\begin{array}{c}\text { Incendi } \\
\text { os } \\
\text { cercan } \\
\text { os }\end{array}$ & $\begin{array}{c}\text { Mal } \\
\text { Man } \\
\text { ejo } \\
\text { fores } \\
\text { tal } \\
\end{array}$ \\
\hline & $\begin{array}{c}\text { VALO } \\
\text { RES }\end{array}$ & 3 & 2 & 2 & 2 & 2 & 2 & 1 & 1 & 1 \\
\hline $\begin{array}{c}\text { Recursos } \\
\text { de } \\
\text { respuesta }\end{array}$ & 10 & 10 & 7 & 7 & 7 & 7 & 7 & 3 & 3 & 3 \\
\hline $\begin{array}{c}\text { Falta } \\
\text { cultura } \\
\text { de } \\
\text { prevenció } \\
\text { n } \\
\end{array}$ & 9 & 9 & 6 & 6 & 6 & 6 & 6 & 3 & 3 & 3 \\
\hline $\begin{array}{c}\text { Falta de } \\
\text { planes de } \\
\text { Gestión } \\
\text { de } \\
\text { Riesgos. }\end{array}$ & 8 & 8 & 6 & 6 & 6 & 6 & 6 & 3 & 3 & 3 \\
\hline $\begin{array}{l}\text { Falta una } \\
\text { política } \\
\text { de } \\
\text { Gestión } \\
\text { de } \\
\text { Riesgos. } \\
\end{array}$ & 8 & 8 & 5 & 5 & 5 & 5 & 5 & 3 & 3 & 3 \\
\hline $\begin{array}{c}\text { acceso a } \\
\text { la } \\
\text { informaci } \\
\text { ón } \\
\end{array}$ & 7 & 7 & 5 & 5 & 5 & 5 & 5 & 2 & 2 & 2 \\
\hline $\begin{array}{l}\text { Organiza } \\
\text { ción } \\
\text { comunita } \\
\text { ria }\end{array}$ & 7 & 7 & 5 & 5 & 5 & 5 & 5 & 2 & 2 & 2 \\
\hline $\begin{array}{l}\text { Hábitos } \\
\text { Tradicion } \\
\text { ales } \\
\text { dañinos } \\
\end{array}$ & 4 & 4 & 3 & 3 & 3 & 3 & 3 & 1 & 1 & 1 \\
\hline $\begin{array}{c}\text { Ubicación } \\
\text { (distancia } \\
\text { ) }\end{array}$ & 3 & 3 & 2 & 2 & 2 & 2 & 2 & 1 & 1 & 1 \\
\hline Pobreza & 3 & 3 & 2 & 2 & 2 & 2 & 2 & 1 & 1 & 1 \\
\hline $\begin{array}{c}\text { Densidad } \\
\text { arbórea }\end{array}$ & 2 & 2 & 2 & 2 & 2 & 2 & 2 & 1 & 1 & 1 \\
\hline $\begin{array}{c}\text { Desarroll } \\
\text { o del } \\
\text { turismo } \\
\end{array}$ & 2 & 2 & 1 & 1 & 1 & 1 & 1 & 1 & 1 & 1 \\
\hline $\begin{array}{c}\text { Accesibili } \\
\text { dad }\end{array}$ & 1 & 1 & 1 & 1 & 1 & 1 & 1 & 0 & 0 & 0 \\
\hline $\begin{array}{c}\text { Propieda } \\
\text { d del } \\
\text { suelo } \\
\end{array}$ & 0 & 0 & 0 & 0 & 0 & 0 & 0 & 0 & 0 & 0 \\
\hline
\end{tabular}

Elaborado: Víctor Espinoza, 2016 
Luego de haber realizado el análisis de las amenazas y vulnerabilidades y aplicado la fórmula de valoración del riesgo se obtuvo el resultado en la tabla 4 . Interpretando esta tenemos 3 niveles de riesgo que se los detalla a continuación.

\section{Riesgo Muy Alto}

Para que el conato de incendio se presente de forma inminente debe presentarse uno de los siguientes factores de vulnerabilidad como el caso de la presencia de piro maniáticos, o la población cercana al bosque tenga bajos recursos para la respuesta, que no se haya trabajado en una cultura de prevención, por ende la falta de elaboración de un plan de gestión de riesgos, y la falta la aplicación de la política pública. Generan un alto riesgo; las "quemas controladas" son un factor que incide mucho en los incendios forestales puesto que la Población cercana a los páramos realiza esta actividad como tradición para la regeneración del pasto, quemas agrícolas, de basura, y los fumadores, vuelve vulnerable al bosque porque las población no está informada o capacitada en la protección del ecosistema y la organización comunitaria no emprende acciones para reducir esta actividad.

\section{Riesgo Medio}

Con respecto a los riesgos medianos, corresponde a la falta de acceso a la información sobre los incendios forestales, la más notables es cuando los niños por intentar cazar conejos de la zona prenden fuego y en muchos de los casos pierde el control o dejan encendido; posteriormente por acción del viento se propaga el fuego. Este caso también se combina con los hábitos tradicionales dañinos que se mantiene en la región andina.

\section{Riesgo Bajo}

Referente al bajo riesgo este se presenta por el grado de pobreza que existe en la zona, la densidad arbórea, el desarrollo del turismo que genera la presencia de mayor personas en el área elevando el nivel de vulnerabilidad y por ende aumentando el riesgo de incendios. $\mathrm{Y}$ por último tenemos la accesibilidad que en este caso por encontrarse en una pendiente superior a $\operatorname{los} 45^{\circ}$ y con vientos fuertes hace que sea difícil contrarrestar los posibles conatos de incendios que se puedan generar en la plantación.

\section{Propuesta del modelo de gestión para la prevención de incendios forestales en función de la problemática existente en la zona y el marco legal del Sistema Nacional de Gestión de Riesgos.}

De acuerdo al análisis que realiza. (Oña Sosa, 2016) en Ecuador el tema de legislación ambiental tiene como punto de partida la constitución de 1983 en la que se reconoce los derechos ambientales como "el derecho a 
vivir en un medio ambiente sano libre de contaminación" más tarde como uno de los iconos constitucionales del Ecuador en la constitución del 2008 se promulga a la naturaleza como sujeto de derechos, obteniendo como resultado que la población tiene derecho a vivir en un ambiente sano y saludable y el Estado como responsable de la conservación y manejo del ambiente garantizando un desarrollo sustentable. Relacionándose directamente con la incidencia de los incendios forestales.

De acuerdo al manual de Gestión de Riesgos de la SNGR en el cual menciona que para abordar el marco legal de la Gestión de Riesgos en el Ecuador es pertinente referirse a los Instrumentos constitucionales y jurídicos que la sustentan. (Secretaría Nacional de Gestión de Riesgos, 2011) - La Constitución de la República del Ecuador

- La Ley de Seguridad Pública y del Estado

- El Reglamento a la Ley de Seguridad Pública y del Estado

- El Código Orgánico de Ordenamiento Territorial Autonomías y Descentralización (COOTAD)

- El Código Orgánico de Planificación y Finanzas Públicas (COPLAFIP)

- Plan Nacional de Desarrollo para el "Buen Vivir" - 20132017

- Ley Orgánica del Sistema Nacional de Compras Públicas.

Estos instrumentos se conviertes en el soporte legal para la gestión de la prevención de los incendios forestales.

El objetivo general de la propuesta del modelo de gestión es establecer directrices para la prevención, control y mitigación de incendios forestales. Para ello se plantean 3 lineamientos con cada uno de las acciones necesarias para el desarrollo de la propuesta de este modelo de gestión de la prevención de los incendios forestales de acuerdo al grafico 3.

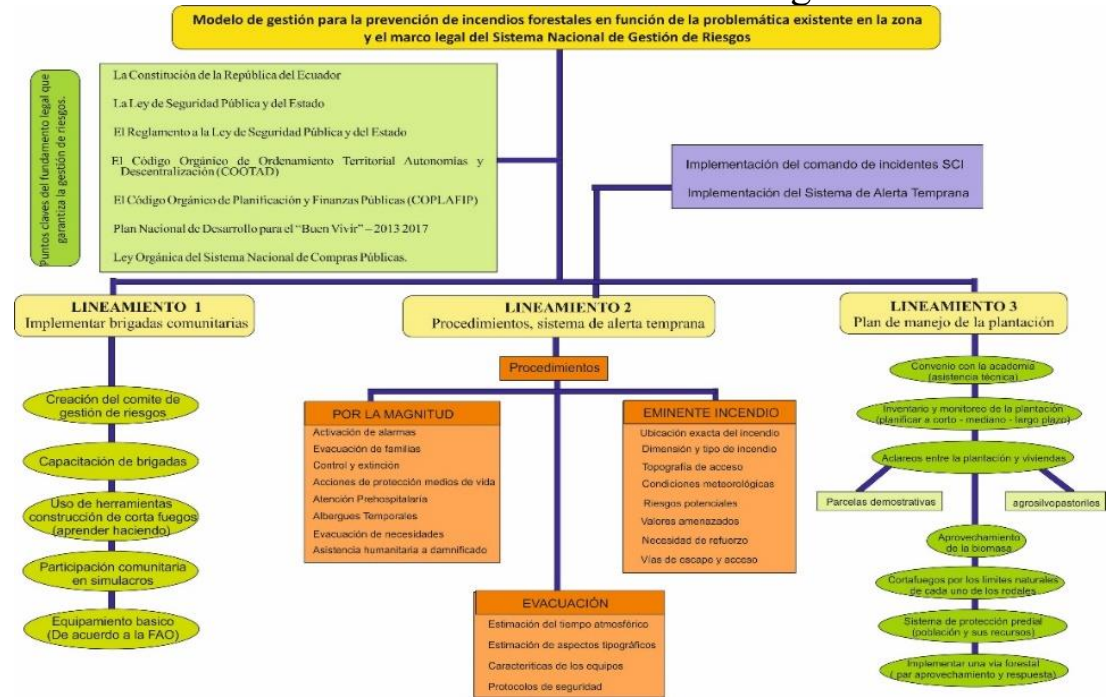

Figura 3. Modelo de Gestión para reducción de los incendios forestales Fuente: Autores 
De acuerdo al grafico xxx nos muestra la estructura del modelo de gestión para la reducción de los incendios forestales donde se detalla cada uno de los lineamientos planteados.

- Implementar procesos de formación de brigadas comunitarias contra incendios, capacitación y entrenamiento para mejorar la respuesta en prevención y atención de incendios en páramos y bosques de altura.

- Diseñar acciones para la prevención y control de conatos de incendios forestales, estableciendo procedimientos y sistemas de alerta temprana para la intervención oportuna.

- Desarrollar un plan de manejo forestal con procedimientos para la prevención y mitigación de incendios forestales.

\section{Conclusion}

De acuerdo al diagnóstico realizado de la situación histórica de los incendios forestales se determina que en el Ecuador no existe un verdadero registro histórico de los incendios forestales. Solamente se cuenta con evidencias de eventos registrados por el proyecto LA RED desde 1972 en el cual la información se registra de forma esporádica hasta el año 2008, por lo que es necesario que cada una de las instituciones responsables asuman con una responsabilidad compartida el seguimiento a cada uno de los eventos.

Como resultado del análisis de riesgos se identificaron 9 amenazas potenciales, 10 vulnerabilidades que se encuentran dentro de la comunidad de Ganquis como en la plantación de Pinus radiata, lo que ha generado el interés y motivación para la participación comunitaria en la prevención y control de los incendios forestales por lo que es necesario que exista una mayor coordinación de acciones entre los propietarios de la plantación y la comunidad que se encuentra aprovechando los recursos existentes en la zona y entre estos recursos la biomasa generada en la plantación.

La implementación de las brigadas comunitarias dependerá exclusivamente del apoyo brindado por los propietarios del plantación, el Gobierno descentralizado de la parroquia de San Juan y la asistencia decisiva de la Secretaria de Gestión de Riesgos como del Ministerio del Ambiente.

\section{Recomendaciones}

Se recomienda difundir la presente propuesta del modelo de gestión con el fin que sea aplicado en otras comunidades y plantaciones forestales con similares condiciones de ubicación geográfica y estructura social comunitaria.

Es importante que a nivel de la Secretaria de Gestión de Riesgos y el Ministerio del Ambiente se estandarice un sistema de registro de incendios forestales con un contenido mínimo de información con el fin de poder realizar estudios mucho más detallados sobre la situación de los incendios 
forestales en la región andina y el grado de afectación que estos generan hacia los medios de vida de las comunidades.

A partir de los análisis y cálculos realizados en esta investigación se ha identificado la necesidad que la academia participe activamente en investigaciones que involucran el impacto de los incendios forestales sobre los recursos suelo, agua, flora y fauna post incendio.

\section{References:}

1. Basantes Carrillo, C. (2011). PROPUESTA PARA LA IMPLEMENTACIÓN DE TURISMO COMUNITARIO. Quito.

2. Castillo, M., Pedernera, P., \& Peña, E. (2003). Incendios Forestales y medio ambiente: una síntesis global. Ambiente y Desarrollo de CIPMA, 10.

3. Centro de Estudios para la Gestión Publica CEGESPU. (2015). Plan de Desarrollo y Ordenamiento Territorial de la Parroquia de San Juan. Riobamba: CEGESPU.

4. Estrategia Internacional para la Reducción de Desastres. (2009). Terminología sobre reducción de riesgos. Roma: UNISDR.

5. FAO. (2007). Situacion de los bosques del mundo 2007. Roma.

6. Guitián Rivera, L. (1999). Los incendios forestales a trave's de la historia: Pervivencias y cambios en el uso del fuego en el noreste peninsular. UNIA.

7. IRg/USAID/OFDALAC. (2006). Curso de operaciones de prevención y control de incendios forestes. Quito.

8. Manta, M., \& León, H. (2004). Los Incendios Forestales en el Perú: Grave Problema por Resolver. Floresta.

9. Oña Sosa, E. V. (2016). Los Incendios Forestales en el área ecológica dentro del Cerro Ilaló, Valle de. Tesís de grado academico para abogada no publicada.

10. Secretaria de Gestión de Riesgos. (2016). Informe Situacional 68. Guaquil: SMEVA.

11. Secretaría Nacional de Gestión de Riesgos. (2011). MANUAL DE GESTION DE RIESGOS PARA EMERGENCIAS Y DESASTRES. Quito: CCSS - SNGR. 